\title{
Uno
}

\section{Pensar en}

la educación

del futuro

DOI: 10.29236/sistemas.n158a6

Pensar en los ciudadanos del futuro.

\section{Resumen}

En la actualidad, muchos aspectos de lo que se ha definido como revolución digital, han sido plataforma de cambios estructurales en los sistemas sociales, económicos, políticos, científicos y por supuesto, educativos de nuestras poblaciones. Resultado de todo ello es la integración de ambientes de ocio, familia, trabajo y educación en una concepción de sujeto de aprendizaje ubicuo y conectado que traduce; en todo lugar, en cualquier momento y de múltiples maneras. El presente artículo propone un análisis de la sociedad actual y los desafios para la educación en un mundo que se transforma a enormes velocidades. En segundo lugar, se considera importante desarrollar una perspectiva crítica del papel que han jugado los observatorios de tendencias de las tecnologías de la educación superior. Finalmente, se ofrece un panorama de las competencias que, a pesar de venirse trabajando desde hace tiempo, continúan siendo problemas actuales y futuros si no se resuelven. Finalmente, se registra un mapa de las competencias del presente que, a juicio de múltiples expertos, es indispensable tener en cuenta para su proyección a futuro.

\section{Palabras clave}

Competencias digitales, observatorios, competencias científicas, Innovación educativa, Educación del futuro. 


\section{Introducción}

Con el cuidado de no ser exclusivista, se puede decir que uno de los factores de mayor importancia de la educación consiste en formar ciudadanos que aportan de manera especializada en la industria, la economía, la política, la ciencia, la cultura, la salud. No contar con sistemas educativos robustos tiende a tener consecuencias de deficiencias, insuficiencias o irregularidades en el aporte de su campo a dichos sectores sociales.

Sin embargo, la educación debe construir juegos complejos de lenguaje sobre el presente con perspectiva histórica. No puede quedarse en la nostalgia del pasado ni saltar desmesuradamente hacia la expectativa de futuro, pues ni pasado ni futuro existen de manera física y formal, y sin embargo juntos son referente fundamental para el presente. En la actualidad, no es sorprendente que todas las tendencias educativas tengan como centro la tecnología digital. Esta se ha convertido en la base de todos los desarrollos de la industria, el entretenimiento, la educación, la política, la economía, la salud, la cultura y otros.

El papel que juega la tecnología en cada uno de estos sectores sociales es fundamental para la construcción de un ecosistema de sociedad del conocimiento y del aprendizaje. Tener claro esto es im-

portante ya que la sociedad contemporánea se encuentra íntimamente articulada con una serie de revoluciones científicas iniciadas en el siglo XVIII. Estas revoluciones son cada vez más complejas en el manejo de la información que fluye en toda la sociedad, del conocimiento que se puede producir con ella y del aprendizaje que implica innovar continuamente las perspectivas del tratamiento de dicho conocimiento. Por lo tanto, no se habla de ciencia ficción cuando se menciona que el ciudadano del siglo XXI deberá tener una formación con una lógica científica contemporánea ${ }^{2}$ compleja.

En dicho contexto, existe un consenso en que el lenguaje que impera actualmente es el dialogo entre industria 4.0 o revolución industrial y Sociedad 5.0. Sin embargo, este tipo de lenguaje plantea relaciones sociales de diferente tipo.

Entre otras; los seres humanos ya no son los actores sociales exclusivos, se encuentra en proceso una diversificación de las relaciones laborales y de transformación empresarial, se consolidan fenómenos crecientes de tercerización en el ecosistema de servicios, es cada vez más clara la emergencia de

Se tiene en cuenta que la lógica científica contemporánea ha abandonado las antiguas peleas entre lo cualitativo y lo cuantitativo, o la mirada epistemológica de las disciplinas cambiándola por una perspectiva holística de pensamientos (computacional, matemático, crítico, creativo, socioemocional etc.) 
empresas con base tecnológica, así como la apertura significativa de unidades y departamentos de Ciencia, Tecnología e Innovación en las organizaciones, reconociendo que el mundo empresarial del siglo XXI ya no funciona sin un núcleo interno de investigación-innovación.

Siempre existen múltiples perspectivas para resolver los problemas; sin embargo, la educación debe integrarse en la aventura de intentarlo y no quedarse petrificada, a riesgo de equivocarse muchas veces(Cano M, 2020). Fortalecer la prospectiva en la educación significa resolver problemas del pasado y fortalecer los procesos de aprendizaje con el propósito de avanzar en la transformación del conocimiento actual hacia el futuro.

\section{Educación del futuro-}

\section{humanos y sociedades del}

\section{futuro}

Pensar el futuro siempre ha sido un imperativo para los sistemas sociales humanos "la predicción de eventos futuros -vital para moverse eficientemente-, es sin duda, la función cerebral fundamental y más común"(Llinás, 2002, p. 25). La pregunta es ¿cómo pensar el futuro en un sector específico como la educación?, ¿es una dificultad o una virtud, su carácter transversal en la sociedad?, es decir que todo sector social <economía, industria, política, saludo, cultura, entretenimiento etc.> se encuentra íntimamente ligado a la educación.
Interesa, en primer lugar, destacar la historia que ha permitido tener una base de reflexión para pensar la educación actual y su prospectiva de futuro. Entre las tendencias que marcaron el escenario se destacan tres metáforas:

En primer lugar, la ya popularmente conocida categoría de "Sociedades del conocimiento" (Misión Internacional de Sabios, 2019; Unesco, 2005), secundada por una idea de sociedades de la información y que luego fue complementada con el concepto de sociedades del aprendizaje.

En segundo lugar, la "Cuarta revoIución industrial." (Nahón, 2018; Schwab, 2016), la cual surgió a medida que crecía nuestro optimismo y efervescencia por las TIC y las posibilidades de transformación digital como actor fundamental en el mejoramiento de sectores sociales, científicos, políticos, económicos y educativos.

En tercer lugar, la "Sociedad 5.0." (Council for Science, Technology and Innovation, 2015; Gallardo, 2020), enfocada en el análisis de vivir en una sociedad altamente digitalizada, sus implicaciones en el desarrollo y prospectiva de la Ciencia, la Tecnología y la Innovación, y el papel que debe jugar la educación para formar los ciudadanos en este contexto.

En torno a estas tres metáforas, han surgido otros análisis y postu- 
ras que describen las implicaciones de la transformación psicológica y social que se gesta a raíz de las revoluciones científico-tecnológicas de los últimos dos siglos y como ello impacta en una noción de formación de sujeto, quien es finalmente el actor central de la educación. Algunos de estos trabajos impactan de manera significativa la reflexión sobre el futuro de la educación ¿cómo y en que formar a las nuevas generaciones?, ¿qué cuidado debemos tener con ellos y cuales son los impactos del tipo de formación que se les ofrece?

En esta franja son importantes los trabajos de Byung-Chul Han(2018; 2017) quien prende alarmas sobre una creciente violencia neuronal padecida de manera cada vez más continua y sistemática por los sujetos contemporáneos: "Las enfermedades neuronales como la depresión, el trastorno por déficit de atención, por hiperactividad (TD$A H)$, el trastorno límite de la personalidad (TLP), o el síndrome de desgaste ocupacional (SDO), definen el panorama patológico de comienzos de este siglo"(2017, p. 7), esta violencia estaría centrada en un principio de positividad producida por fenómenos de superproducción, supercomunicación y superrendimiento, en los cuales el individuo se encuentra en cotidiana competencia con las máquinas automatizadas.

La industria 4.0 generara nuevos empleos indudablemente. Sin em- bargo, para aquellos seres humanos que realizan las tareas para las cuales se diseñan las máquinas producto de la revolución, la lucha es y será dramática y está perdida a mediano y largo plazo. ¿cómo puede la educación contribuir a resolver la gran pandemia del desempleo por falta de formación especializada en América Latina?

En la misma dirección pero con diferentes consecuencias, la revolución científica digital ha impactado la economía y la industria de manera disruptiva. Estos sectores sufren un proceso de transformación estructural y aún no se identifica un horizonte de estabilidad. En dicho sentido, la industria ya no requiere al sujeto de obediencia que se formó en el sistema educativo de las revoluciones anteriores. Ahora requiere formar un sujeto de emprendimiento y autónomo. Para Han, el ciudadano de la arcaica retórica moderna ya no existe más, y ahora emerge un "individuo proyecto" que tiene como misión autogestionarse a sí mismo en una sociedad del rendimiento que le solicita exigirse hasta el límite. ¿Se encuentra la educación en perspectiva de formar sujetos que no necesariamente se consideran empleados en el sentido moderno del término?

En otro sentido, los últimos años, han tomado relevancia los análisis de Yuval Harari (2015b, 2015a). De acuerdo con este autor, nos encontramos en el bucle de las revolucio- 
nes científicas que comenzaron en el siglo XVIII, y es conveniente pensar en lo que ocurre actualmente, no como una, sino como múltiples revoluciones tecnológicas, que se transforman permanentemente.

Pero especialmente, Harari obliga a pensar interrogantes como: ¿hemos ganado felicidad con el transcurrir de la historia? ¿Podemos hacer algo para influir en los siglos futuros?, ¿Qué lecciones hemos aprendido de las revoluciones anteriores? En su opinión, cada revolución ha sido un ecosistema autónomo de representaciones simbólicas que ha generado una serie de comunidades imaginadas. La serie de revoluciones digitales que ocurren en la actualidad no parece ser la excepción.

En este extraño y plurirevolucionario proceso, Harari plantea que están ocurriendo transformaciones radicales en la manera como se definen los seres humanos. Cada vez nos acostumbramos a vernos menos como una unidad de "cuerpo y alma" y más como una "colección de mecanismos bioquímicos que está constantemente supervisada y guiada por una red de algoritmos electrónicos"(Harari, 2015a).

Realmente en el campo de la medicina, existen múltiples experiencias que pueden ser referencia de esta transición, y ello ha estado desplazándose de manera veloz al mundo del Fitness con las aplicaciones móviles(Nike, 2021).
A ese proceso, Harari lo denomina como dataismo; un conjunto de información altamente detallada almacenada en diferentes sistemas de información sobre cada uno de los seres humanos y que tiene como función principal retroalimentar diferentes sistemas de algoritmos que día a día, avanzan en la misión de ganarse la confianza de los seres humanos. El trofeo en la batalla es la imposición de una perspectiva en la toma de decisiones sobre la propia existencia humana cotidiana, expresada en las acciones que se realizan en el entorno familiar, laboral, de entretenimiento, educativo, de salud, económico etc. ¿Esta el sistema educativo universitario formando en competencias de análisis, comprensión y protección del uso y manejo de datos?

¿Qué puede sustituir los deseos y las experiencias como origen de todo sentido y autoridad? En 2016, únicamente una candidata está sentada en la sala de espera de la historia, aguardando la entrevista de trabajo. Esta candidata es la información. La religión emergente más interesante es el dataísmo, que no venera ni a dioses ni al hombre: adora los datos(Harari, 2015a).

Estos dos autores tienen consenso sobre la transformación profunda que ha implicado el cambio de mundo provocado por las tecnologías digitales y su integración con el ecosistema social, político, científico, económico y cultural. El problema de competencia y aprendi- 
zaje desborda completamente el tradicional discurso del sistema educativo y se instala en la cotidianidad de los seres humanos bajo el paradigma de computación ubicua en un contexto de aprendizaje a lo largo de la vida (en cualquier lugar, en cualquier momento y de múltiples maneras) en una carrera de aprendizaje y formación continua, sin límite formal.

El único inconveniente es que la noción de cambio se desarrolla a una velocidad tan grande que difícilmente se tiene certeza de las condiciones de mundo en la siguiente década, o incluso en el siguiente quinquenio. En consecuencia, es importante apostar por el soporte que realizan los observatorios y sin embargo, lograr leer entrelineas sus recomendaciones para tener clara la sutil diferencia entre tendencia y predicción, en un discurso que se ha vuelto cotidiano y popular en miles de empresas, tecnológicas, de gestión del conocimiento y de innovación educativa.

\section{Un análisis a los \\ observatorios de tendencias y predicciones educativas}

Indudablemente, el panorama presentado es fuerte y desafiante para un alto porcentaje de la población mundial contemporánea. El campo educativo siempre ha estado en medio de esta tensión que significa formar sujetos útiles a las sociedades en que viven, pero también con competencias críticas y creativas que permitan encontrar siempre mejores soluciones de futuro para todos los sectores sociales de la población.

Actualmente, los observatorios de tendencias en innovación son el mejor lugar para entender el desarrollo de las expectativas educativas. También permiten analizar la manera desigual en que dichas tendencias se consolidan. Estos observatorios no son entendidos como tradicionalmente se manejaba este término en el mundo científico moderno tradicional (un espacio de observación y registro de la realidad), sino que juegan un papel de construcción de la realidad, es decir, se fabrican predicciones de la transformación del presente de cara al futuro. Es la metáfora contemporánea de lo que se entendió como tecnociencia desde la mitad del siglo XX (Echeverría \& González, 2009; Latour, 2005).

Un ejemplo significativo de esta nueva forma de tratar la predicción de futuro en el campo educativo son los reportes del "proyecto Horizon". Comenzaron a ser realizados en el 2004 por el consorcio New Media Consortium (NMC), y a partir de 2017 han sido continuados por la empresa EDUCASE(EDUCASE, 2021). El reporte Horizon ha sido un referente importante para analizar de manera estratégica la relación entre tecnologías emergentes desarrolladas en la industria y su posible vinculación con procesos educativos. 
El reporte se estructura a partir de tres elementos clave: 1. Tendencias clave que aceleraran la adopción de tecnología en la educación superior, 2- Desarrollos clave en tecnología educativa, y 3. Desafíos que impiden la adopción de tecnología en la educación superior. La vinculación de dicha tecnología proveniente de la industria y proyectada al mundo educativo, es analizada a corto, mediano y largo plazo(Fuerte, 2019).

Sin embargo, durante los 16 años en que ha sido publicado, el reporte nunca ha tenido un análisis retrospectivo del impacto de las propuestas de tendencias generadas cada año. Algunos especialistas del mundo educativo mencionan la necesidad de complementar estos análisis con un "guiño de la historia": Es que sin un guiño a la historia de estas predicciones, es difícil ver el informe como mucho más que una aceptación de la narrativa de que "todo cambia (Watters, 2019, 2021).

Lo anterior, genera múltiples interrogantes y sobre todo, tiende a crear una alta percepción de pesimismo sobre la apuesta que realiza un país o una institución con la vinculación de una tecnología en sus procesos educativos. En consecuencia, se vende la predicción como tendencia y quien la adquiere corre el grave riesgo de adquirir tan solo una ilusión de x o y compañía que quería posicionar determinados productos. ¿cómo se puede ver este fenómeno en el histórico de las predicciones del informe horizon? Expertos del Observatorio de Innovación Educativa del Instituto Tecnológico de Monterrey, analizan las expectativas generadas en México alrededor del aprendizaje adaptativo, formulado como una tendencia en el informe horizon de 2015. Concluyen que ocurrió algo similar a lo expuesto por Waters: se da un estrangulamiento de la historia que no pasa desapercibido con el transcurrir del tiempo.

Esta tendencia apareció por primera vez en el Informe Horizon como una tendencia importante a seguir en 2015. Desde entonces, el tiempo predicho para la adopción se ha movido de cuatro a cinco años en la edición de 2015, a un año o menos en 2017 y de dos a tres años en el informe del año pasado. Este año, sin embargo, el aprendizaje adaptativo ni siquiera apareció en la lista de desarrollos(Fuerte, 2019).

¿Qué ha pasado con este caso y muchos otros que a lo largo de estos años (2004-2018) han sido publicados como tendencias en el proyecto Horizon y finalmente no aparecen en el mundo educativo? o se convierten en una retórica circular (Downes, 2019) Para el caso del aprendizaje adaptativo, los expertos creen que aún no se han desarrollado todas las herramientas necesarias para trasladar este modelo al sistema educativo, y en aquellas donde se ha intentado ma- 
terializar se ha generado altas inversiones para las instituciones en materia recursos, tiempo y dinero. Algo que en las instituciones educativas de América Latina, tanto públicas como privadas, difícilmente se podría dar.

Adicionalmente, esta nueva revolución científica del mundo contemporáneo es un ecosistema con alta volatilidad y disrupción en la forma de entender los sentidos acerca del sujeto, la sociedad y los sectores sociales que orientan la política pública. Son diferentes lenguajes que no hay que suplantar sino más bien articular (por ejem: el reconocimiento de la diferencia de tiempos entre la producción industrial y los procesos de aprendizaje, investigación e innovación): Ciertamente necesitamos volver atrás y aprender de nuestros errores. Es crucial que cada vez que leamos o escuchemos acerca de una nueva predicción sobre el futuro de la educación o de una nueva tendencia, volvamos a los pronósticos que se hicieron en el pasado $y$ analicemos qué les sucedió (Fuerte, 2019). En el siguiente cuadro histórico del informe se puede leer la disrupción en la formulación de tendencias que aparecen y desaparecen a lo largo de la historia sin mayor explicación.

Finalmente, es importante destacar que la empresa EDUCASE, recogió las críticas realizadas al modelo del informe y en su reporte, versión 2020, modificó su estructura, aclarando que cómo toda predicción, tiene altas probabilidades de error. Vincula una perspectiva histórica de pasado y presente como elementos clave en el análisis de la predicción realizada a futuro, y en lugar de la predicción desnuda que se había mantenido en la pasada década, se ofrecen datos, evidencias y escenarios para que los tomadores de decisiones en la política pública gubernamental e institucional, tengan elementos más concretos para pensar un futuro de las tecnologías susceptibles de aplicar en la educación(Brown et al., 2020b).

\section{Tabla 1: Histórico de tendencias informe Horizon 2004-2018.}

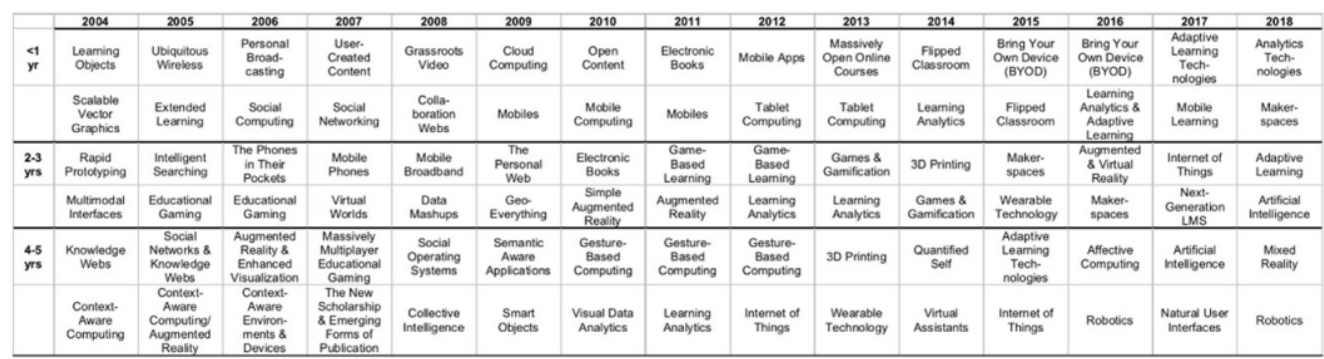

Tomado de: Watters, A. Consultada en: 2018-08-16-horizon.png $(2408 \times 658)$ 
3. La formación de las nuevas generaciones de profesionales: Juegos de supervivencia, juegos de reinvención

\subsection{Una educación global/local con perspectiva de ciudadanía científica}

De acuerdo con el contexto anteriormente expuesto, pensar los procesos de formación de las futuras generaciones de profesionales significa proyectar un ecosistema en el que se involucran diversas perspectivas. Es cada vez más importante dar el lugar a la tecnología sin perder de vista los propósitos centrales de la formación de ciudadanía.

Existe una enorme gama de propuestas de prospectiva con relación a lo que acontecerá al mundo educativo según la perspectiva social y/o el campo de conocimiento desde donde se observe. Las conclusiones generadas por el Foro Internacional de Innovación Educativa (FIIE), son importantes ya que recogen una mirada a la problemática nacional, debido a la participación de varias universidades del país.

Se considera importante identificar variables de contexto como: 1 . Los cambios demográficos que determinan el crecimiento de la población global, 2. El uso y conservación de los recursos naturales y energéticos, 3. La mitigación de los efectos del cambio climático, 4. Tendencia de la población mundial a congregarse cada vez más densamente en los centros urbanos, 5 . Una mayor autonomía de la gobernanza regional, Y 6 . Una creciente preocupación por la salud pública. (Prieto-Martinez, 2021)

En la misma dirección, la versión 2020 del informe Horizon, se reinventa y propone el análisis de tendencias sobre la base de contextos sociales, tecnológicos, económicos, de educación superior y políticos.

Adicionalmente, plantea la necesidad de complementar la relación entre tecnologías emergentes en educación, con la instalación de escenarios de reflexión sobre el futuro de la educación superior misma, y plantea cuatro escenarios posibles para el futuro de la educación (Tabla 2).

\subsection{Una educación a futuro con perspectiva histórica: \\ Actualizar, fortalecer y adquirir nuevas competencias}

Así mismo, otros análisis de orden general permiten ofrecer una perspectiva histórica y de contexto acerca de la importancia de actualizar algunas, fortalecer otras, y adquirir nuevas competencias en clave de tecnociencias. Este escenario se configura como una base para que las futuras generaciones pueden navegar en este nuevo archipiélago de revoluciones científicas que acompañan el surgimiento de las sociedades contemporáneas. 


\begin{tabular}{|l|l|}
\hline ESCENARIO & DESCRIPCIÓN \\
\hline Crecimiento & $\begin{array}{l}\text { la próxima década de educación superior es una caracterizado } \\
\text { por un progreso significativo, con un crecimiento próximo de } \\
\text { aumentos en adultos y estudiantes remotos, expansión de } \\
\text { cursos y planes de estudio en línea, y certificación profesional } \\
\text { y programas de microcredenciales. }\end{array}$ \\
\hline Restricción & $\begin{array}{l}\text { la eficiencia y la sostenibilidad son la guía } \\
\text { valores sociales en este futuro de la educación superior, con } \\
\text { los alumnos se abren caminos más rápidos y eficientes } \\
\text { hasta la finalización y las instituciones aprovechan el poder de } \\
\text { datos y análisis para una mayor precisión en el diseño de } \\
\text { experiencia del alumno y protección del regreso de la } \\
\text { institución en inversión. }\end{array}$ \\
\hline Colapso & $\begin{array}{l}\text { la educación superior, tal como la conocemos, ha cerrado, } \\
\text { principalmente por razones económicas (en aumento costos, } \\
\text { financiamiento decreciente), reemplazado por un nuevo } \\
\text { sistema de educación que prioriza las necesidades del } \\
\text { mercado laboral } \\
\text { y la adquisición de habilidades discretas sobre programas y } \\
\text { departamentos incapaces de proporcionar un retorno de la } \\
\text { inversión. }\end{array}$ \\
\hline Transformación & $\begin{array}{l}\text { ocurren varias transformaciones dramáticas } \\
\text { en la educación superior durante la próxima década, provocó } \\
\text { principalmente por el cambio climático y los avances en digital } \\
\text { tecnología. Los alumnos disfrutan de una matrícula más } \\
\text { flexible y opciones de personalización de títulos, mientras que } \\
\text { las instituciones explorar modelos de redes cooperativas y } \\
\text { buscar formas de reducir el costo de la educación }\end{array}$ \\
\hline
\end{tabular}

Fuente: (Brown et al., 2020b).

Para Colombia como muchos otros países en vías de desarrollo, el futuro de la educación se encuentra comprometido con la necesidad de fortalecer las competencias básicas a saber: lectura, escritura y aritmética: La naturaleza cambiante del trabajo está convirtiendo las competencias básicas de lectura, escritura y aritmética en habilidades necesarias para sobrevivir y para manejarse en la vida cotidiana (...) Millones de niños que viven en países de ingreso bajo y mediano asisten a la escuela durante cuatro o cinco años y aun así no adquieren las competencias básicas de lectura, escritura y aritmética. (World Bank, 2019, p. 138).

Muchos de esos niños crecen y llegan al ciclo profesional universitario con vacíos de competencias básicas, hecho que compromete el proyecto de formar en competencias más complejas y especializadas. 
Tabla.3 Estándares de formación inicial de competencias TIC para profesores

\begin{tabular}{|c|c|c|c|c|c|c|c|c|c|c|}
\hline $\begin{array}{l}\text { Categorías de } \\
\text { Competencias }\end{array}$ & 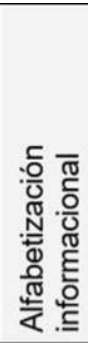 & 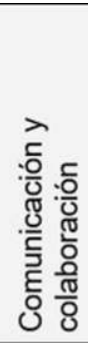 & 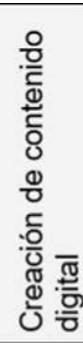 & 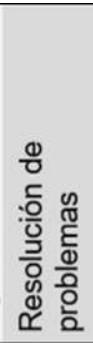 & 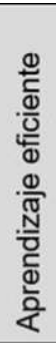 & 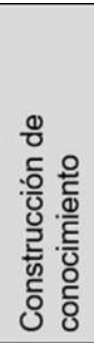 & 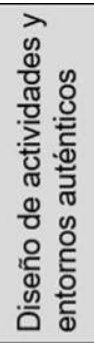 & 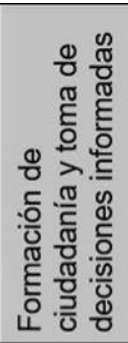 & 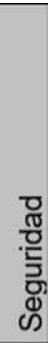 & 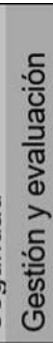 \\
\hline INTEF (2017) & $\mathrm{x}$ & $x$ & $\mathrm{x}$ & $x$ & & & & & $x$ & \\
\hline ISTE (2016) & & $x$ & & $x$ & $x$ & & $x$ & $x$ & & \\
\hline \multicolumn{11}{|l|}{ UNESCO TIC (2011) } \\
\hline & & & & $x$ & $x$ & $\mathrm{x}$ & & & & \\
\hline \multirow[t]{2}{*}{ UNESCO MIL (2011) } & $x$ & & & & & & & & & \\
\hline & & & & $x$ & & & & $x$ & $\mathrm{x}$ & \\
\hline $\begin{array}{l}\text { MEN Colombia } \\
(2013)\end{array}$ & & $\mathrm{x}$ & & $\mathrm{x}$ & $\mathrm{X}$ & $\mathrm{X}$ & & & & $\mathrm{x}$ \\
\hline
\end{tabular}

Fuente: (Leal-Urueña \& Rojas-Mesa, 2018a)

En ese mismo orden, es importante actualizar y fortalecer las políticas y lineamientos gubernamentales e institucionales sobre competencias digitales. El país cuenta con una propuesta desactualizada sobre el tema (Calderón, Gina; Buitrago, Betty; Acevedo, María; Tobon, 2013; Leal-Urueña \& Rojas-Mesa, 20 18a; Ministerio De Educacion Nacional, 2008) Al respecto, internacionalmente existe un panorama claro sobre procedimientos y estándares de competencias digitales que bien valdría la pena que aparecieran en las propuestas de formación de las universidades nacionales y se proyectarán a las instituciones de educación básica y media (Tabla 3).

Así mismo, se encuentra la necesidad de profundizar en competen- cias para el desarrollo de aprendizaje en educación virtual e hibrida, que involucra una formación intensiva en competencias digitales '-(Burbules, 2012; Cope \& Kalantzis, 2021; Rojas-Mesa, Julio Ernesto; Leal-Urueña, 2017; RojasMesa, 2013; Sangrá et al., 2019; Van Den Beemt \& Diepstraten, 2016) La pandemia que atraviesa el mundo (COVID) ha dejado claro que si bien, muchas instituciones educativas no tienen claro aún como vincular en su oferta académica esquemas de educación virtual o mixta, lo cierto es que esta se ha convertido en un huésped permanente en todo proyecto educativo de colegios y universidades (Palacios, 2021)

Un campo de competencia que nunca pasara de moda y que al 
contrario, cobra cada vez más relevancia a pesar de lo complejo que significa estar en dicha dimensión, tiene que ver con la participación del talento humano en Ciencia, Tecnología e Innovación, conocido bajo la noción de competencias científicas.

Los enfoques de investigación científica, funcionan bajo un esquema organizado en dos dimensiones desde la mitad del siglo XX, La primera: Big Science o Macro ciencia, funciona en el orden global, y la segunda: tecnociencia, opera en escenarios empresariales, civiles y ciudadanos locales (Echeverría \& González, 2009)

De cara a no perder el referente de la dimensión global macro científi$\mathrm{ca}$, algunas instituciones educativas con énfasis en la investigación, deberán fortalecer mucho más las competencias de Ciencia-Tecnología e Innovación de futuros profesionales con miras a hacer parte de diferentes escenarios de "Big Science"; grandes proyectos de interés mundial, en los cuales participan científicos de diferentes partes del mundo (Scarrà \& Piccaluga, 2020)

América Latina, debido a su escaso desarrollo científico, ha tenido una participación estatal marginal, sin embargo, de manera particular, algunos de los científicos formados en países de la región participan en diferentes proyectos de este tipo y son referente para nuevas genera- ciones de profesionales con grandes capacidades científicas. Colombia en particular tiene referentes importantes en varios campos científicos, en diferentes lugares del mundo - (Colombia.co, 2021; Space-School \& -Colombia, 2021). Los profesionales formados en esta dimensión no se quedan necesariamente en el país, pero cumplen una función importante en las nuevas perspectivas de CTI, haciendo parte de un fenómeno mundial denominado circulación global de competencias.

En la dimensión local, la materialización social los procesos científicos se conoce como tecnociencia y surge gracias a la revolución digital, la participación activa del sector empresarial en procesos de investigación-innovación y al fortalecimiento de una metáfora de política pública en Investigación, Desarrollo e Innovación, conocida como I+D+I. Los profesionales que se formen en esta dimensión deben tener fuertes competencias de ciudadanía científica y competencias del siglo XXI. En un mundo que cambia de manera veloz, es apenas natural que surja una industria que se transforma al mismo ritmo y consecuentemente un talento humano formado en dicha lógica (World Bank, 2019).

\subsection{Relaciones entre educación y tecnologías emergentes: \\ Sociedades $\mathrm{N}^{\circ}{ }^{\circ}$}

Como se ha mencionado a lo largo de este escrito, es una condición 
Tabla 4 Cuadro de tendencias sobre educación para el futuro

\begin{tabular}{|c|c|c|}
\hline TENDENCIA & INFORME/REPORTE & IMPLICACIONES \\
\hline $\begin{array}{l}\text { Educación virtual, Learning, } \\
\text { modelos mixtos. }\end{array}$ & $\begin{array}{l}\text { Informe Horizon-2020 } \\
\text { de EDUCASE (Brown et } \\
\text { al., 2020a), Reporte de } \\
\text { innovación educativa- } \\
2019-2020 \text {, del TEC de } \\
\text { Monterrey (Palacios, } \\
\text { 2021) }\end{array}$ & $\begin{array}{l}\text { Formación en } \\
\text { competencias } \\
\text { digitales, en } \\
\text { docentes y } \\
\text { estudiantes }\end{array}$ \\
\hline $\begin{array}{l}\text { Formas alternativas de desarrollo } \\
\text { de la oferta académica } \\
\text { universitaria }\end{array}$ & $\begin{array}{l}\text { Informe Horizon-2020 } \\
\text { de EDUCASE, Reporte } \\
\text { de innovación } \\
\text { educativa-2019-2020, } \\
\text { del TEC de Monterrey, } \\
\text { Informe 2020-Iniciativa } \\
\text { los futuros de la } \\
\text { educación-UNESCO } \\
\text { (Haste, Helen; Chopra, } \\
\text { 2021) }\end{array}$ & $\begin{array}{l}\text { Formación en } \\
\text { competencias } \\
\text { problémicas, y } \\
\text { micro } \\
\text { aprendizaje. }\end{array}$ \\
\hline $\begin{array}{l}\text { La educación universitaria y la } \\
\text { industria, cada vez más } \\
\text { enfocadas en los ODS }\end{array}$ & $\begin{array}{l}\text { Informe MIRADAS-2019 } \\
\text { de la OEI, Informe 2020- } \\
\text { Iniciativa los futuros de } \\
\text { la educación-UNESCO } \\
\text { (Collective, 2021) }\end{array}$ & $\begin{array}{l}\text { Formación en } \\
\text { competencias } \\
\text { socioemocionales } \\
\text { y de ciudadanía } \\
\text { científica }\end{array}$ \\
\hline $\begin{array}{l}\text { Analíticas de aprendizaje y } \\
\text { Dataísmo }\end{array}$ & $\begin{array}{l}\text { Informe Horizon-2020 } \\
\text { de EDUCASE, Índice } \\
\text { mundial de innovación- } \\
2020 \text { (Business, 2020), } \\
\text { Reporte-2020, del } \\
\text { Instituto de Tecnología } \\
\text { educativa- Open } \\
\text { University-Londres, } \\
\text { Informe 2020-Iniciativa } \\
\text { los futuros de la } \\
\text { educación-UNESCO }\end{array}$ & $\begin{array}{l}\text { Formación en } \\
\text { competencias } \\
\text { para el análisis } \\
\text { de datos }\end{array}$ \\
\hline $\begin{array}{l}\text { Bienestar, salud mental, } \\
\text { protección de datos y privacidad }\end{array}$ & $\begin{array}{l}\text { Informe Horizon-2020 } \\
\text { de EDUCASE, Informe } \\
2020 \text {-Iniciativa los } \\
\text { futuros de la educación- } \\
\text { UNESCO, }\end{array}$ & $\begin{array}{l}\text { Formación en } \\
\text { competencias de } \\
\text { Ciberseguridad }\end{array}$ \\
\hline IA y Robótica & $\begin{array}{l}\text { Informe Horizon-2020 } \\
\text { de EDUCASE, Índice } \\
\text { mundial de innovación- } \\
2020 \text {, Reporte de } \\
\text { innovación educativa- } \\
2019-2020 \text {, del TEC de } \\
\text { Monterrey, Informe } \\
2020 \text {-Iniciativa los } \\
\text { futuros de la educación- } \\
\text { UNESCO }\end{array}$ & $\begin{array}{l}\text { Formación en } \\
\text { competencias de } \\
\text { pensamiento } \\
\text { computacional y } \\
\text { STEM }\end{array}$ \\
\hline Ecologías de aprendizaje & $\begin{array}{l}\text { Reporte de innovación } \\
\text { educativa-2019-2020, } \\
\text { del TEC de Monterrey } \\
\text { (Palacios, 2021), }\end{array}$ & $\begin{array}{l}\text { Formación en } \\
\text { competencias de } \\
\text { aprendizaje } \\
\text { autónomo }\end{array}$ \\
\hline
\end{tabular}

Fuente: Elaboración propia 
esencial de la sociedad contemporánea entender la tecnología como un actor equivalente a los seres humanos en dimensiones de impacto en el mundo individual, social y ambiental, de interacción y comunicación en términos de lenguaje y de colaboración en términos de trabajo y aprendizaje. Muchas de las tendencias y predicciones presentadas a continuación, encierran esta nueva lógica social.

En la tabla 4 podemos identificar las tendencias que marcan un quehacer fundamental en la educación superior de cara al presente y futuro de nuestras sociedades. Así mis$\mathrm{mo}$, se registran las organizaciones que han apostado por estas tendencias.

En dicho sentido, es bueno saber que por nuestra condición cerebral, continuaremos profundizando en sistemas y tecnologías de predicción de nuestro futuro cada vez más complejos. El cuadro pone sobre la mesa algunas variables de los desarrollos tecnológicos en educación e investigación educativa. Sin embargo, no alcanzan a dimensionar otras tantas que indudablemente condicionan el flujo y resultado de cada una de estas tendencias.

Es importante, trabajar en el análisis local sobre variables de infraestructura, competencias básicas, cultura organizacional, capacidad institucional, para cruzarlas con la matriz presentada anteriormente.
Estos resultados permitirán establecer líneas base discontinuas a lo largo y ancho del país educativo, y permitirán proyecciones personalizadas (institucionales) sobre la educación presente que debe estar necesariamente integrada a las tecnologías y cuales desarrollos se pueden potenciar a futuro.

\section{Algunas conclusiones}

La presente reflexión ha permitido pensar el futuro de la educación desde un paradigma de conocimiento contemporáneo, analizando el tipo de sociedades que se esta conformando y los paradigmas sociales, científicos y tecnológicos desde donde se está construyendo.

Ha sido importante y lo será en el futuro cada vez más, entender la manera como funcionan los observatorios de tendencias y predicciones, así como comprender la diferencia. Ello proveerá a las organizaciones gubernamentales, educativas y expertos en educación de elementos más precisos para generar apuestas por tecnologías en educación de acuerdo con los recursos contados.

El escrito propone una ruta de análisis que consiste en pensar la ciudadanía científica como el camino más práctico y eficaz para que a futuro la población nacional pueda sintonizarse en contextos cada vez más complejos de información, producción de conocimiento, diseño de experiencias de aprendizaje 
y tecnologías integradas. Ello permitirá que los estudiantes no conciban los sistemas educativos universitarios nacionales como su único y exclusivo territorio de formación, desafiándolos a configurar ecosistemas de aprendizaje ubicuo cada vez más especializados y personalizados.

La educación universitaria colombiana del futuro, más eficaz será aquella que sea capaz de convertir el discurso de competencias de ciudadanía de siglo XXI, en una cultura organizacional de su institución y una forma didáctica concreta del aprendizaje de sus estudiantes en todos los campos de conocimiento.

Estamos conectados global y localmente. Por ahora, la universidad colombiana ha convertido estos paradigmas en mera retórica pedagógica pero no científica o de investigación contundente. Es una tarea fundamental para las universidades.

\section{Referencias}

Brown, M., Mccormack, M., Reeves, J., Brooks, D. C., Grajek, S., Alexander, B., Bali, M., Bulger, S., Dark, S., Engelbert, N., Gannon, K., Gauthier, A., Gibson, D., Gibson, R., Lundin, B., Veletsianos, G., \& Weber, N. (2020b). 2020 EDUCAUSE Horizon Report TM Teaching and Learning Edition Teaching and Learning Edition Thank You to Our Horizon Report Sponsors Platinum Partner Platinum Partner. https://www.educause. edu/horizon-report-2020
Burbules, N. C. (2012). El aprendizaje ubicuo y el futuro de la enseñanza. Encuentros Sobre Educación, 13 (2), 3-14.

Business, S. J. C. of. (2020). Índice mundial de innovación 2020.

Calderón, Gina; Buitrago, Betty; Acevedo, María; Tobon, M. (2013). Competencias tic para el desarrollo profesional docente.

www.mineducacion.gov.co

Cano M, J. J. (2020, March 1). Sandbox: Revindicate failure as the foundation of learning. EDUNINE 2020 4th IEEE World Engineering Education Conference: The Challenges of Education in Engineering, Computing and Technology without Exclusions: Innovation in the Era of the Industrial Revolution 4.0, Proceedings.

https://doi.org/10.1109/EDUNINE4 8860.2020 .9149512

Collective, C. W. R. (2021). Learning to become with the world: Education for future survival.

https://learningportal.iiep.unesco.o rg/en/library/learning-to-becomewith-the-world-education-for-future -survival

Colombia.co. (2021). Colombianos Trabajando En Ciencias Naturales| Marca País Colombia. Online Site. https://www.colombia.co/pais-co lombia/talento-de-colombia/6-co lombianos-destacados-en-el-mun do-por-su-trabajo-en-ciencias-natu rales/

Cope, B., \& Kalantzis, M. (2021). Pedagogies for Digital Learning 25 Oct 20. Dear 2017, 1-19. 
Council for Science, Technology and Innovation, G. of J. (2015). Report on The 5 th Science and Technology Basic Plan Council for Science , Technology and Innovation Cabinet Office, Government of Japan.

Downes, S. (2019). Horizon Report Preview $2019 \sim$ Stephen Downes. Online Site.

https://www.downes.ca/cgi-bin/pa ge.cgi?post $=69215$

Echeverría, J., \& González, M. I. (2009). La teoría del actor-red y la tesis de la tecnociencia. Arbor, 185 (738), 705-720.

https://doi.org/10.3989/arbor.2009. 738n1047

EDUCASE. (2021). EDUCAUSE Homepage|EDUCAUSE. Online. https://www.educause.edu/

Fuerte, K. (2019). El Informe Horizon mira hacia atrás por primera vez. Online Site.

https://observatorio.tec.mx/edunews/informe-horizon-2019-mirahacia-atras-por-primera-vez

Gallardo, S. (2020). Nuevas tecnologías, desde una visión antropológica. Revista SISTEMAS, 154, 24-28.

https://doi.org/10.29236/sistemas. n154a4

Han, Bull-Chung. (2018). Hiperculturalidad. Visión Electrónica, 12(2), 339-343.

https://revistas.udistrital.edu.co/ind ex.php/visele

Han, Byung-Chul. (2017). La sociedad del cansancio. Han, Byung-Chul,. https://www.amazon.com/-/es/Byu
ng-Chul-Han-ebook/dp/B074Z PCGD4/ref=sr_1_7? mk_es_US $=A ̊ M A ̌ Z ̌ O ̃ N \tilde{N} \& \bar{d}$ illd $=1$ \&keywords $=B$ yung-Chul+Han\&qid=1612643852 \&sr $=8-7$

Harari, Y. N. (2015a). Homo Deus. In Book. ePubLibre.

Harari, Y. N. (2015b). De animales a dioses. Breve historia de la humanidad. 1-27.

Haste, Helen; Chopra, V. (2021). The futures of education for participation in 2050 : educating for managing uncertainty and ambiguity.

https://unesdoc.unesco.org/ark:/48 223/pf0000374441

Latour, B. (2005). Reassembling the social . An Introduction to Actor-Network-Theory. In Book.

Leal-Urueña, L. A., \& Rojas-Mesa, J. E. (2018b). Ecología para la formación inicial de profesores a partir de los affordances de las TIC. Tecné Episteme y Didaxis: TED, 44. https://doi.org/10.17227/ted.num4 4-8986

Llinas, R. R. (2002). El cerebro y el mito del yo; el papel de las neuronas en el pensamiento y comportamiento humanos. In Editorial Norma (Ed.), Libro (Sexta).

https://www.amazon.es/El-Cere bro-Mito-del-Yo/dp/958046798645 180.pdf\%0A

https://hdl.handle.net/20.500.1238 0/245180\%0A

http://dx.doi.org/10.1016/j.jsames. 2011.03.003\%0Ahttps://doi.org/10. 1016/j.gr.2017.08.001\%0A http://dx.doi.org/10.1016/j.precamr es.2014.12.0 
Ministerio De Educacion Nacional. (2008). República de Colombia Apropiación de TIC en el Desarrollo Profesional Docente. Ministerio de Educación Nacional, 1-20.

Misión Internacional de Sabios. (2019). Colombia hacia una sociedad del conocimiento. In Informe de la misión internacional de sabios 2019 por la educación, la ciencia, la tecnología y la innovación.

Nahón, A. E. (2018). Redefinición del en red " en la cuarta revolución industrial. Apertura: Revista de Innovación Educativa, 10, 149163.

https://doi.org/http://dx.doi.org/10.1 8381/Ap.v10n1.1140

Nike. (2021). Nike Training Club App. Sitio Web.

https://www.nike.com/es/ntc-app

Palacios, E. (2021). Contenido.

https://observatorio.tec.mx/edunews/reporte-innovacion-educati va-2020

Prieto-Martinez, L. D. (2021). Megatendencias y tecnologías emergentes que afectan la dirección y ritmo de cambio en la Educación Superior | Linkedln. Online Site.

https://www.linkedin.com/pulse/me gatendencias-y-tecnolog $\% 25$ C3\%25ADas-emergentes-queafectan-prieto-mart $\% 25$ C $3 \% 25 A D$ nez/?trackingld=GBcc9zRP9Yf1U s2myvJinw\%3D\%3D

Rojas-Mesa, Julio Ernesto; LealUrueña, L. A. (2017). Affordance: constructo para la comprensión y transformación del aprendizaje en contextos interculturales. TED: Tecné, Episteme y Didaxis, 42.

Rojas-Mesa, J. E. (2013). Educación virtual: del discurso teórico a las prácticas pedagógicas en la educación superior colombiana. Universidad Nacional de Educación a Distancia (España). Facultad de Educación. Departamento de Teoría de la Educación y Pedagogía Social.

http://e-spacio.uned.es/fez/eserv/ tesisuned:Educacion-Jerojas/Do cumento.pdf

Sangrá, A., Raffaghelli, J. E., \& GuitertCatasús, M. (2019). Learning ecologies through a lens: Ontological, methodological and applicative issues. A systematic review of the literature. British Journal of Educational Technology.

https://doi.org/10.1111/bjet.12795

Scarrà, D., \& Piccaluga, A. (2020). The impact of technology transfer and knowledge spillover from Big Science: a literature review.

https://doi.org/10.1016/j.technovati on.2020.102165

Schwab, K. (2016). The Fourth Industrial Revolution. In Book.

Space-School, \& -Colombia. (2021). Colombianos en la NASA - Colombia Space School. Online Site. https://www.colombiaspaceschool. org/colombianos-en-la-nasa/

Unesco, I. D. La. (2005). Hacia las sociedades del conocimiento. In Editorial UNESCO.

https://doi.org/ISBN 92-3-304000-3 
Van Den Beemt, A., \& Diepstraten, I. (2016). Teacher perspectives on ICT: A learning ecology approach. Computers and Education, 92-93, 161-170.

https://doi.org/10.1016/j.compedu. 2015.10.017

Watters, A. (2019). The 100 Worst EdTech Debacles of the Decade. Online Site. http://hackeducation.com/2019/12/ 31/what-a-shitshow

Watters, A. (2021). A Horizon Report History. Blog.

http://horizon.hackeducation.com/

World Bank. (2019). Naturaleza cambiante del trabajo. In World Bank Publications (Ed.), report. www.bancomundial.org

Julio Ernesto Rojas Mesa. Doctor en Teoría de la Educación y Pedagogía Social UNEDEspaña. Profesor del Doctorado en Educación de la Universidad Santo Tomás. Director línea Educación, cultura y sociedad. Líder grupo Investigación educativa, Clasificación A ScienTI-COL-2019.

Código ORCID: https://orcid.org/0000-0002-2237-3727

Google Scholar: https://scholar.google.com/citations?user=e0Xtbe8AAAAJ\&hl=es 